\title{
http://bjas.journals.ekb.eg \\ Diffusion Kurtosis Imaging of Acute Infarction in Comparison with Routine Diffusion
}

M.M.Refaat, A.E.Shaalan, M.A.Attia

Radio diagnosis Dept., Faculty of Medicine, Benha Univ., Benha, Egypt

E-Mail:Mohamed.adel.3000@gmail.com

\begin{abstract}
Stroke is a major global health problem with an estimated incidence rate of around 15 million new cases each year worldwide. It could be classified into ischemic and hemorrhagic that account for approximately $85 \%$ and $15 \%$ of all cases, respectively.The aim of the work is to perform a systematic review of the literature regardingdiffusion kurtosis Imaging and routine diffusion in ofAcute infarction. We will shed the light on the results of using both imaging techniques, and showing advantages, disadvantages of each technique. Endovascular therapy is highly beneficial compared with tissue plasminogen activator alone, particularly at extended time of stroke onset. It has been shown that prospective classification according to infarction core volume by using diffusion-weighted magnetic resonance (MR) imaging and clinical criteria is associated with a higher likelihood of favorablethrombectomy outcome.
\end{abstract}

\section{Introduction}

Stroke is a major global health problem with an estimated incidence rate of around 15 million new cases each year worldwide. It could be classified into ischemic and hemorrhagic that account for approximately $85 \%$ and $15 \%$ of all cases, respectively [1].

Diffusion-weighted imaging (DWI) provides information about the amount of random Brownian movements of water molecules. DWI has rapidly gained popularity. Although it produces poor spatial resolution compared with T2WI, it is useful as a supplementary technique in drawing attention to areas of suspicion, hence increasing the accuracy of morphologic T2WI. [2] In a meta-analysis, the pooled sensitivity and specificity of DWI was $62 \%$ and $90 \%$ respectively [3].

DWI was a groundbreaking advance in stroke imaging in the 1990s. Restricted diffusion was once thought to be pathognomonic of cerebral infarction, but with experience this binary approach to infarction has become outdated. The extent of tissue damage is often quite heterogeneous within ischemic brain, having so-called restricted diffusion. Improved differentiation between ischemic and infarcted tissue for purposes of identifying potentially salvable brain tissue has become of particular importance as intraarterial and IV reperfusion techniques continue to improve [4].

Lu et al. [5] confirmed that DKI provides an expedient approach for refining the heterogeneous DWI lesion that is associated with graded metabolic derangement, which is promising for improving the infarction core definition and ultimately helping to guide stroke treatment.

\section{Stroke}

Stroke is a major global health problem with an estimated incidence rate of around 15 million new cases each year worldwide. Stroke is a leading cause of fatality and long-term disability. It is currently the third leading cause of death preceded only by heart disease and cancers. Fatality rate increases progressively in the post-ischemic stroke period with an estimated rate of $33 \%$ and $80 \%$ fatalities in the first year and 10 years, respectively [6].

\subsection{Definition of stroke}

Stroke is defined as an "acute neurologic dysfunction of vascular origin with sudden (within seconds) or at least rapid (within hours) occurrence of symptoms and signs corresponding to the involvement of focal areas in the brain [7] confirmed by brain CT and/or MRI in baseline conditions [8].

\section{Definition of CNS infarction}

Central nervous system (CNS) infarction is brain, spinal cord, or retinal cell death attributable to ischemia, based on:

- Pathological, imaging, or other objective evidence of cerebral, spinal cord, or retinal focal ischemic injury in a defined vascular distribution; or

- Clinical evidence of cerebral, spinal cord, or retinal focal ischemic injury based on symptoms persisting _24 hours or until death, and other etiologies excluded [9].

\subsection{Stroke risk factors}

A risk factor for ischemic stroke is a characteristic in an individual that increased risk of ischemic stroke compared with an individual without that characteristic [10]. Epidemiological studies have advanced our understanding of stroke risk factors and clinical trials have demonstrated effective interventions to decrease stroke risk by modifying risk factors. Stroke risk factors are classified as traditional and novel and may be further divided into modifiable and non-modifiable [11].

\subsubsection{Hypertension}


There is overwhelming evidence that prolonged elevated BP increases the risk of developing either a fatal or nonfatal stroke [12].

\subsubsection{Cigarette smoking}

Cigarette smoking is closely related to the top 4 leading causes of death in the United States, which are heart disease, cancer, cerebrovascular disease, and chronic obstructive pulmonary disease. Moreover, Pearson et al. [13] has been determined smoking as a biologically plausible independent predictor of stroke after controlling for known risk factors of stroke.

\subsubsection{Diabetes mellitus}

The risk of stroke is increased between 2.5 and 3.6 fold in individuals with diabetes [14], and the risk of fatal stroke is higher in those with a higher $\mathrm{HbA1c}$ at diagnosis [15].

\subsubsection{Cardiac diseases}

The presence of cerebrovascular disease is strongly associated with the presence of symptomatic and asymptomatic cardiac disease [16].

\subsubsection{Dyslipidemia}

Hyperlipidemia is an important modifiable risk factor for cardiovascular disease. Three prospective studies in men subsequently showed increases in ischemic stroke rates at higher levels of total cholesterol, especially for levels above 240 to 270 $\mathrm{mg} / \mathrm{dl}$ [17].

\subsubsection{Obesity and Body Fat Distribution}

However, several studies have suggested that abdominal obesity, (waist circumference $102 \mathrm{~cm}$ in men and $88 \mathrm{~cm}$ in women), rather than general obesity, is associated with the risk of stroke [18].

\subsubsection{Metabolic Syndrome}

The metabolic syndrome is a substantial predictor of coronary heart disease, cardiovascular disease, and all-cause mortality. Furthermore, obesity is an important component of the metabolic syndrome and is associated with major health risk factors, poor health status, and lower life expectancy [19].

\subsubsection{Oral Contraceptive (OCs) Use}

The increased stroke risk associated with the use of OCs is based on early studies with high dose preparations ( $\square 50 \mathrm{ug}$ of estradiol [20].

\section{Diffusion-weighted imaging of acute infarction}

Diffusion-weighted imaging (DWI) provides information about the amount of random Brownian movements of water molecules. Protons are very mobile in normal water-rich glandular tissue, but restricted in their movement in densely packed water-poor tissue such as tumor areas, which contain many hydrophobic cell membranes [21].
DWI has rapidly gained popularity. Although it produces poor spatial resolution compared with T2WI, it is useful as a supplementary technique in drawing attention to areas of suspicion, hence increasing the accuracy of morphologic T2WI. Furthermore, an interesting correlation seems to exist between the degree of diffusion restriction and tumor aggressiveness (Gleason score) [2].

In a meta-analysis, the pooled sensitivity and specificity of DWI was $62 \%$ and $90 \%$ respectively [3]. In another meta-analysis, the accuracy, sensitivity and specificity of T2WI combined with diffusion were $90 \%, 85 \%$ and $95 \%$ respectively, compared to $77.5 \%, 65 \%, 90 \%$ when using T2WI alone .

\subsection{Principles of diffusion-weighted MR imaging}

DWI exploits the random motion of water molecules. In a totally unrestricted environment, water movement would be completely random, a phenomenon otherwise known as Brownian motion or free diffusion [22]. Within biologic tissues, the movement of water is not completely random, but rather, is impeded by interaction with tissue compartments, cell membranes, and intracellular organelles. For purposes of simplification, water movement in tissues may be categorized as intravascular, intracellular, or extracellular Fig (1) [23].

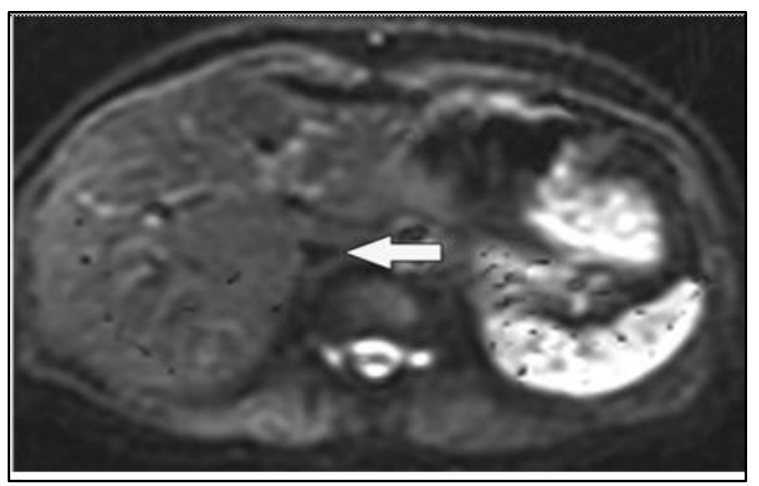

Fig (1) Axial diffusion-weighted image $(b=0$ $\mathrm{sec} / \mathrm{mm} 2$ ) obtained in a 60-year-old woman shows a signal void within the inferior vena cava (arrow).

\subsection{Diffusion of water molecules in tissue}

DWI explores the random motion of water molecules in the body. Water molecules held in a container outside the body are in constant random Brownian motion. This uninhibited motion of water molecules is free diffusion. By contrast, the movement of water molecules in biologic tissues is restricted because their motion is modified and limited by inter actions with cell membranes and macromolecules.

In biologic tissue, the DWI signal is derived from the motion of water molecules in the extracellular space, the intracellular space, and the 
intravascular space. Not surprisingly, given a unit time, water molecules in the intravascular space will have a greater diffusion distance because of blood flow than those in the extracellular and intracellular spaces. Clearly, the contribution of intravascular water diffusion to the measured DWI signal can vary among tissues. In tumors showing increased vascularity, the contribution of intravascular water diffusion to the MR signal may account for a significant proportion. [24].

\subsection{Measuring water motion (apparent diffusion) using DWI}

Stejskal and Tanner [25] described an MR experiment that could be applied to the detection and quantification of water diffusion in vivo. They adapted a standard T2-weighted spin-echo sequence by applying a symmetric pair of diffusion-sensitizing (bipolar) gradients around the $180^{\circ}$ refocusing pulse. That approach is now the basis of many DWI sequences in clinical use today. Static molecules acquire phase information from the first diffusion gradient, but information will be rephased by the second diffusion gradient without a significant change in the measured signal intensity. By comparison, moving water molecules acquire different phase information from the first gradient, but because of their motion, their signal will not be completely rephased by the second gradient, thus leading to a signal loss (figure 21). Hence, the motion of water molecules is detected as attenuation of the measured signal intensity at DWI. The degree of water motion has been found to be proportional to the degree of signal attenuation.

The sensitivity of the DWI sequence to water motion can be varied by changing the gradient amplitude, the duration of the applied gradient, and the time interval between the paired gradients. On clinical MR scanners, the diffusion sensitivity is easily varied by changing the parameter known as the "b value," which is proportional to these three factors. When the b value is changed, it is usually the gradient amplitude, rather than the duration or time intervals between gradients, that is altered. Water molecules with a large degree of motion or a great diffusion distance (e.g., within the intravascular space) will show signal attenuation with small $b$ values (e.g., $b=50-100$ $\mathrm{s} / \mathrm{mm}^{2}$ ).Unlike, large $\mathrm{b}$ values (e.g., $\mathrm{b}=1,000$ $\mathrm{s} / \mathrm{mm}^{2}$ ) are usually required to perceive slowmoving water molecules or small diffusion distances because these show more gradual signal attenuation with increasing $b$ values [22].

DWI yields qualitative and quantitative information that reflects tissue cellularity and cell membrane integrity, which complements the morphologic information obtained by conventional MR imaging [22].

\subsection{Interpretation of DWI}

By performing DWI using different $b$ values, quantitative analysis is possible. This analysis is usually performed at a push of a button on the scanner or workstation that results in the calculation of the apparent diffusion coefficient (ADC). One simplified method of visualizing this process is by considering the signal attenuation of a tissue with increasing $b$ values. By plotting the logarithm of the relative signal intensity of the tissue on the $y$-axis against the $b$ values on the $x$ axis, a line can be fitted through the plots (exponential function). The slope of the line describes the ADC. Not surprisingly, the fit can be improved by using more $\mathrm{b}$ values to reduce the error in ADC calculation [22].

The ADC is independent of magnetic field strength and can overcome the effects of T2 shinethrough, thus allowing more meaningful comparison of results. The ADC is calculated for each pixel of the image and is displayed as a parametric map. By drawing regions of interests on these maps, the ADCs of different tissues can be derived. Areas of restricted diffusion in highly cellular areas show low ADC values compared with less cellular areas that return higher ADC values. However, it is simplistic to assume that the attenuation of the signal intensity with increasing $b$ values occurs linearly in tissues [22].

\subsection{Technical considerations regarding routine diffusion in acute infarction}

Diffusion weighted imaging (DI) has now become a routine technique in the magnetic resonance (MR) protocols for the evaluation of stroke patients. In 1986, the intravoxel incoherent motion and diffusion Imaging was introduced by Le Bihan et al. [24]. However only by the mid1990 s the method became a routine in clinical practice because of its demanding MR engineering requirements especially high performance magnetic field gradients. Its primary application has been in the investigation of acute stroke patients where it provides unique information about the physiologic state of ischemic tissue. It also helps in distinguishing irreversible ischemic lesions from potentially reversible lesion characterized by vasogenic oedema Fig (2).

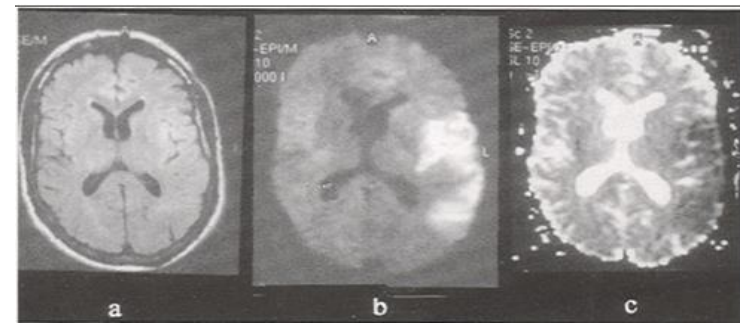

Fig (2) 54 year old male patient with sudden onset of weakness on the right half of he body.

\subsubsection{Reliability of DWI in acute stroke}


New thrombolytic and neuro protective therapies are being developed to treat acute ischemic infarction. Good results have been shown if the treatment is undertaken within the first 3 hours. Hence early detection of the ischemic region has gained a lot of importance. After a stroke, detection of hypoattenuation in CT scan and hyperintensity on $\mathrm{T} 2$ weighted images require a substantial increase in tissue water. For infarction imaged within 6 hours of stroke onset, reported sensitivities are $38 \%$ to $45 \%$ for CT scan and $18 \%$ to $46 \%$ for MR imaging. Diffusion weighted imaging has shown to be highly sensitive (88$100 \%)$ and specific $(86 \%-100 \%)$ in the detection of hyperacute and acute infarction [26].

Schellinger et al. [27] have shown that DWI is superior to MR imaging, first and second CT in the examination of patients with acute stroke within 24 hours of presentation. The superiority is most clearly demonstrated when the imaging is performed in less than or equal to 6 hours following presentation. After 24 hours, DWI and ADC maps help in distinguishing between acute, sub acute and chronic lesions. This is of great help especially in older patients who have T2 hype rintensities that may be indistinguishable from acute lesions in T2 and FLAIR images.

False negative DWI lesions has been reported for lacunar brain stem or deep grey matter infarcts and in regions of decreased perfusion in perfusion MR imaging. Many of these lesions are picked up in the follow up DWI. False positive DWI lesions with restricted diffusion can be seen in cerebral abscess (due to high viscosity) and in tumor (due to dense cellularity). Differentiation can be made by viewing routine $\mathrm{T} 1, \mathrm{~T} 2$ weighted and post contrast images [28].

\subsubsection{Reversibility}

Although the area identified as hyperintense in DWI is often labeled as infarct this may not be true. This area may show evidence of energy failure and cell regulation defect, however, the cells might not have progressed to ultimate death. Hence there is a definite role to salvage even this so called "infarcted area" [29].

\subsubsection{Transient Ischemic Attacks (TIA)}

DWI demonstrates ischemic abnormalities in nearly half of clinically defined TIA patients. The lesions are usually less than $15 \mathrm{~mm}$ in maximal diameter. The percentage of patients with positive DWI lesion increases with increasing total symptom duration. The compromised vascular territory detected on diffusion imaging and the clinical symptoms correlate. In nearly half the patients, the diffusion MRI changes may be fully reversible, while in the remainder the findings herald the development of a parenchymal infarct despite transient, clinical symptoms [26].

\subsubsection{Correlation of DWI with clinical outcome}

It has been seen in earlier studies that a combination of DWI images with NIHSS (National Institute of Health Stroke scale score) may predict clinical recovery of cortical infarcts than any of the factors taken alone. Statistically significant correlation has been found between the acute diffusion MR lesion volume and both acute and chronic neurological assessment tests. Patients with perfusion deficits larger than the diffusion MR lesion volume had worse clinical outcome compared to those patients who had early reperfusion Fig (3).

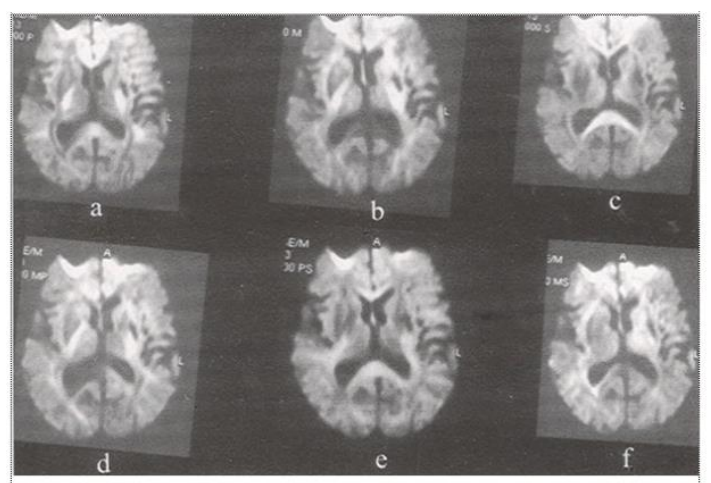

Fig(3) Anisotropic nature of diffusion in the brain. Axial diffusion-weighted images (b value$1000 \mathrm{~s} / \mathrm{mm} 2)$.

\section{Diffusion kurtosis imaging of acute infarction \\ Diffusion imaging, which is based on} brownian motion of water molecules, has been in clinical use for more than 25 years. Originally focused on stroke imaging, the modality has evolved rapidly, gaining numerous clinical applications in neuroradiology and beyond. Diffusion-tensor imaging (DTI) is a more recent application of diffusion imaging and is predicated on determining the directionality of diffusion. DTI has provided additional information on the microstructure of the brain and has been proved to be a particularly elegant, noninvasive method of displaying the anatomic features of the white matter tracts [4].

Gaussian distribution is a mathematic model describing the normative distribution of a given population conforming to the wellknown bell curve. In reality, the complex intracellular and extracellular in vivo environment causes the diffusion of water molecules to deviate considerably from this pattern [4].

In probability theory and statistics, alteration of a normative pattern of distribution is known as kurtosis. Diffusion kurtosis imaging (DKI) is an attempt to account for this variation to provide a more accurate model of diffusion and to capture the nongaussian diffusion behavior as a reflective marker for tissue heterogeneity [30]. 
DKI was applied exclusively for brain imaging [31]. However, Iima et al. [32] have shown the feasibility of applying DKI at multiple extracranial sites. Therefore, body radiologists may benefit through a better understanding of the major concepts of DKI Fig (4).

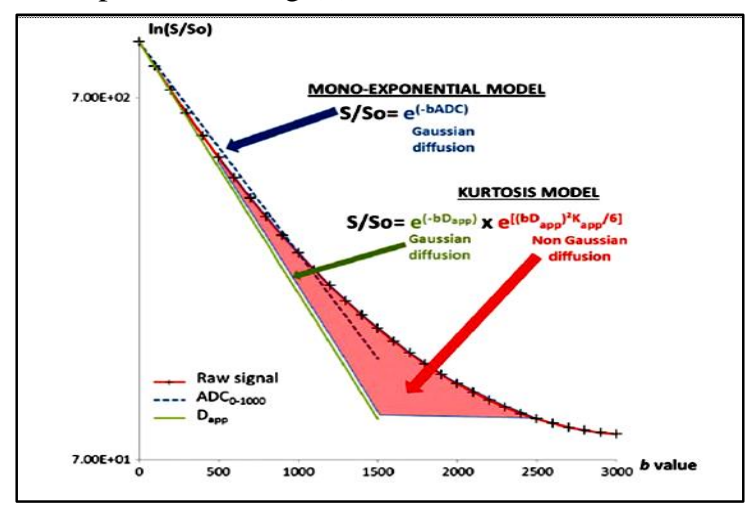

Fig(4) Comparison of diffusion signal intensity decay plots for monoexponential model, reflecting solely Gaussian diffusion, and kurtosis model, reflecting both Gaussian and non-Gaussian diffusion components.

\subsection{Acquisition and postprocessing considerations \\ 4.1.1 Image acquisition}

DKI is acquired using a standard DWI sequence available on current-generation scanners, albeit incorporating ultrahigh $b$-values. Whereas standard DWI requires acquisition of only two bvalues for the calculation of ADC, DKI requires acquisition of at least three distinct $b$-values given an additional unknown variable (Kapp) within the formula [24].

In practice, Rosenkrantz et al. [33] suggested acquiring more than three $b$-values, including at least two b-values both above and below 1000 $\mathrm{s} / \mathrm{mm} 2$, to facilitate the successful capture of the non-Gaussian behavior. If the maximal b-value is not high enough, then the deviation of the curvature of the SI decay plot away from Gaussian will not be captured, and the ability of the sequence to measure non-Gaussian diffusion behavior decreases substantially.

Compared with the brain, tissues in the body generally exhibit faster signal decays at increasing b-values, as well as faster T2 decays. Moreover, larger-sized torso coils have less ideal receptivity and geometry in comparison with head coils. Therefore, the optimal choice of maximal b-value for DKI in body applications is lower than in the brain. Indeed, non-Gaussian behavior of the SI decay plot can be successfully captured at a maximal b-value of 1500-2000 s/mm2 in body imaging. On this basis, a maximal b-value in this range may be appropriate for DKI body applications [34].
As breath-hold imaging is associated with significantly reduced SNR for DWI, either respiratory-triggered or free-breathing acquisitions are needed. Using the minimal possible echo time also helps raise SNR, which is facilitated at ultrahigh b-values through high-performance gradient systems. Use of a 3T system, when available, substantially improves SNR as well. Even with these considerations, SNR may still be insufficient. Post-hoc corrections of "noise floor" effects in the signal remain challenging, although some simple techniques have been proposed for clinical practice [35].

Quentin et al. [36] reported performing kurtosis assessment of the full multidirectional diffusion tensor, described as diffusion kurtosis tensor imaging (DKTI). This approach acquires diffusion images in at least 15 different directions in order to assess the anisotropic directionality of diffusion and kurtosis behavior.

Finally, although not a parameter routinely manipulated by radiologists, the diffusion time, DT, indicating the time between onsets of the two lobes of the diffusionencoding gradient, also impacts kurtosis estimates, given the greater physical diffusion distances probed with increasing DT. A short DT is advised in order to be sensitive to obstacles to non-Gaussian water diffusion that occur at short length scales and that influence Kapp, as well as to mitigate water exchange between tissue compartments that occurs at longer DT. Kapp increases as DT increases from a short DT, reaching a peak value at an intermediate DT, and subsequently decreases with further increases in DT, ultimately approaching zero. In comparison, Dapp is relatively less sensitive to DT length. Shorter DT may be achieved by minimizing TE times, which is facilitated through use of monopolar diffusion-encoding schemes and stronger gradient rise times [37].

\section{Comparison between routine diffusion and diffusion kurtosis imaging of acute infarction}

Berkhemer et al. [38] have convincingly demonstrated endovascular therapy to be highly beneficial compared with tissue plasminogen activator alone, particularly at extended time of stroke onset. To minimize hemorrhage risk and futile recanalization, the infarction core volume is often used as one of the imaging exclusion criteria for endovascular therapy.

It has been shown that prospective classification according to infarction core volume by using diffusion-weighted magnetic resonance (MR) imaging and clinical criteria is associated with a higher likelihood of favorable thrombectomy outcome. With the advancement of more effective endovascular treatment options for late recanalization, it has become increasingly important to properly identify the extent of the 
infarction core to evaluate the treatment effect and to ultimately better guide patient care [39].

Diffusion-weighted imaging (DWI) is sensitive in depicting acute ischemia and has been used for imaging the irreversibly damaged infarction core. Several penumbral assessment paradigms have been practiced, built on the assumption that the lesion on DWI images approximates the infarction core, including perfusion-DWI, MR angiographyDWI, and clinical-DWI mismatches [40].

While DWI is highly sensitive to ischemic insult, its specificity in depicting the infarction core has been questioned. It has been demonstrated that the regions of lowered apparent diffusion coefficient (ADC) experience graded metabolic derangement and include not only the ischemic core but also potentially salvageable penumbral tissue [41].

Yamada et al. [42] have documented DWI reversibility following recanalization, even in some cases of large lesions on DWI images. Unfortunately, the severity of ADC change could not differentiate potentially salvageable lesion at DWI from irreversibly damaged infarction core, and new imaging approaches are needed Fig (5).

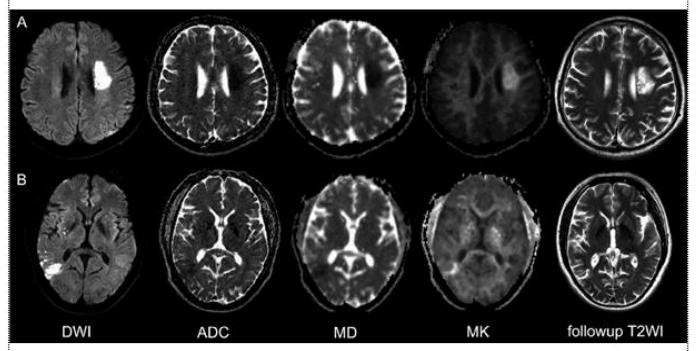

Fig (5) Two representative cases with acute stroke. A, DWI in a 43-year-old man with right-limb weakness for 12 hours.

$\mathrm{B}, \mathrm{DWI}$ in a 53-year-old man with dizziness and vomiting for 12 hours.

\section{Summary}

Endovascular therapy is highly beneficial compared with tissue plasminogen activator alone, particularly at extended time of stroke onset. It has been shown that prospective classification according to infarction core volume by using diffusion-weighted magnetic resonance (MR) imaging and clinical criteria is associated with a higher likelihood of favorablethrombectomy outcome.

Diffusion-weighted imaging (DWI) is sensitive in depicting acute ischemia and has been used for imaging the irreversibly damaged infarction core. Several penumbral assessment paradigms have been practiced, built on the assumption that the lesion on DWI images approximates the infarction core, including perfusion-DWI, MR angiographyDWI, and clinical-DWI mismatches.

The routine DWI analysis assumes that water molecules follow a Gaussian diffusion profile. This assumption, strictly speaking, applies only to the case of unrestricted diffusion and is crude for modeling in vivo diffusion measurement.

The kurtosis-based lesion is smaller than the DWI-based lesion; the kurtosis lesion was shown not to be responsive to early reperfusion, while the kurtosis-diffusion lesion mismatch often reverses following recanalization. This suggests that the kurtosis-based lesion captures the most severely injured portion of the DWI-based lesion, while the part of the DWI-based lesion without kurtosis abnormality appears to have less injury.

\section{References}

[1] F Rincon and RL. Sacco, Secondary Stroke Prevention. J Cardiovasc Nurs, Vol. 23, PP. 34-41, 2008.

[2] T Hambrock, C Hoeks, C Hulsbergen-van de Kaa, Prospective assessment of prostate cancer aggressiveness using 3-T diffusion-weighted magnetic resonance imaging-guided biopsies versus a systematic 10-core transrectal ultrasound prostate biopsy cohort. Eur Urol, Vol. 61(1), PP. 177-84, 2012.

[3] C Jie, L Rongbo, T Ping. The value of diffusion-weighted imaging in the detection of prostate cancer: a meta-analysis. Eur Radiol, Vol. 24 (8) , PP. 1929-41, 2014.

[4] AJ Steven, J Zhu and ER. Melhem, Diffusion kurtosis imaging: An emergening technique for evaluating the microstructural environment of the brain. AJR202, PP. W26-W33, 2014.

[5] D Lu, Y Jiang, Y Ji and IY. Zhou, Evaluation of diffusion kurtosis imaging of stroke lesion with hemodynamic and metabolic MRI in a rodent model of acute stroke. American Journal of Roentgenology, Vol. 210(4) , PP. 720-727, 2018.

[6] MJ Alberts and B. Ovbiagele, Current strategies for ischemic stroke prevention: role of multimodal combination therapies. J Neurol, Vol. 254, PP. 1414-1426, 2007.

[7] JL Hinkle and MM. Guanci, Acute Ischemic Stroke Review. J Neurosci Nurs, Vol. 39(5), PP. 285-310, 2007.

[8] A Tuttolomondo, $\mathrm{R}$ Di Sciacca, D Di Raimondo, A Serio, G D'Aguanno, S La Placa, et al., Plasma levels of inflammatory and thrombotic/fibrinolytic markers in acute ischemic strokes: relationship with TOAST subtype, outcome and infarct site. J Neuroimmunol, Vol. 215(1-2), PP. 84-89, 2009.

[9] RL Sacco, SE Kasner, JP Broderick, LR Caplan, JJ Connors, A Culebras, et al., An Updated Definition of Stroke for the 21st Century: A Statement for Healthcare Professionals From the American Heart Association/American Stroke Association. Stroke, Vol. 44(7), PP. 2064-2089, 2013. 
[10] GJ. Hankey, Potential new risk factors for ischemic stroke what is their potential? Stroke, Vol. 37, PP. 2181-2188,2006.

[11] JR. Romero, Prevention of ischemic stroke: overview of traditional risk factors. Curr Drug Targ, Vol. 8(7), PP. 794-801, 2007.

[12] LE Fields, VL Burt, JA Cutler, J Hughes, EJ Roccella and P Sorlie, The burden of adult hypertension in the United States 1999 to 2000: a rising tide. Hypertension, Vol. 44, PP. 398-404, 2004.

[13] TA Pearson, SN Blair, SR Daniels, RH Eckel, JM Fair, SP Fortmann, et al., AHA Guidelines for Primary Prevention of Cardiovascular Disease and Stroke: 2002 Update: Consensus Panel Guide to Comprehensive Risk Reduction for Adult Patients Without Coronary or Other Atherosclerotic Vascular Diseases. American Heart Association Science Advisory and Coordinating Committee. Circulation, Vol. 106, PP. 388-391, 2002.

[14] A Arboix, A Rivas, L García-Eroles, L de Marcos, J Massons and M. Oliveres, Cerebral infarction in diabetes: Clinical pattern, stroke subtypes, and predictors of in-hospital mortality. BMC Neurol, Vol. 5, PP. 9, 2005.

[15] RJ Stevens, RL Coleman, AI Adler, Risk factors for myocardial infarction case fatality and stroke case fatality in type 2 diabetes: UKPDS 66. Diabetes Care, Vol. 27(1), PP. 201-207, 2004.

[16] LB Goldstein, R Adams, MJ Alberts, Culebras A, DeGraba TJ, Gorelick PB, Guyton JR, Hart RG, Howard G, Kelly-Hayes M, Nixon JV and Sacco RL. A Guideline From the American Heart Association/American Stroke association Stroke Council: Cosponsored by the Atherosclerotic Peripheral Vascular Disease Interdisciplinary Working Group; Cardiovascular Nursing Council; Clinical Cardiology Council; Nutrition, Physical Activity, and Metabolism Council; and the Quality of Care and Outcomes Research Interdisciplinary Working Group. Stroke, Vol. 37, PP. 1583-1633, 2006.

[17] A Kagan, JS Popper and GG Rhoads, Factors related to stroke incidence in Hawaii Japanese men. The Honolulu Heart Study. Stroke, Vol. 11, PP. 14-21, 1980.

[18] YM Song, J Sung, G Davey Smith and S. Ebrahim, Body mass index and ischemic and hemorrhagic stroke: a prospective study in Korean men. Stroke, Vol. 35, PP. 831-836, 2004.

[19] AH Mokdad, BA Bowman, JP. Koplan, The continuing epidemics of obesity and diabetes in the United States. JAMA, Vol. 286, PP. 1195 1200,2001 .

[20] PC Hannaford and PR Croft, Oral contraception and stroke: evidence from the Royal College of General Practitioners' Oral
Contraception Study. Stroke, Vol. 25, PP. 935942, 1994.

[21] DM Somford, JJ Futterer, T Hambrock, Diffusion and perfusion MR imaging of the prostate. Magn Reson Imaging Clin N Am, Vol. 16(4), PP. 685-95, 2008.

[22] DM Koh, DJ Collins, Diffusion-Weighted MRI in Body: Applications and Challenges in Oncology. AJR, Vol. 188, PP. 1622-1635, 2007.

[23] DM Patterson, AR Padhani, DJ Collins. Technology insight: water diffusion MRI: A potential new biomarker of response to cancer therapy. Nat Clin Pract Oncol, Vol. 5(4), PP. 220-233, 2008.

[24] D Le Bihan. Intra-voxel incoherent motion imaging using steady state free precession. Magn. Reson. Med, Vol. 7, PP. 497-505, 1988.

[25] EO Stejskal, JE Tanner. Spin diffusion measurements: spin-echo in the presence of a time dependent field gradient. J Chem Phys, Vol. 42, PP. 288 -292, 1965.

[26] CS Kidwell, JL Saver, J Mattiello, Thrombolytic reversal of acute human cerebral ischemic injury shown by diffusion/perfusion magnetic resonance imaging. Ann Neurol, Vol. 47, PP. 462-469, 2000.

[27] PD Schellinger, JB Fiebach, W Hacke. Imaging-based decision making in thrombolytic therapy for ischemic stroke: present status. Stroke, Vol. 34(2) , PP. 575583, 2003.

[28] H Ay, F Buonanno. Schaefer P et al. Clinical and diffusion - weighted imaging haracteristics of an identifiable subset of TIA patients with acute infarction. Proceedings of the 24th American Heart association. International Conference on Stroke and Cerebral Circulation. Nashville, TN, 1999.

[29] J Fiehler, JB Fiebach, A Gass, Diffusionweighted imaging in acute stroke-a tool of uncertain value? Cerebrovasc Dis, Vol. 14, PP. 187 - 196, 2002.

[30] EX Wu, MM Cheung. MR diffusion kurtosis imaging for neural tissue characterization. NMR Biomed, Vol. 23, PP. 836-848, 2010.

[31] E Fieremans, JH Jensen, JA Helpern. White matter characterization with diffusional kurtosis imaging. NeuroImage, Vol. 58, PP. 177-188, 2011.

[32] M Iima, K Yano, M Kataoka, Quantitative non-Gaussian diffusion and intravoxel incoherent motion magnetic resonance imaging: differentiation of malignant and benign breast lesions. Invest Radiol, Vol. 50, PP. 205-211, 2015.

[33] AB Rosenkrantz, EE Sigmund, A Winnick, Assessment of hepatocellular carcinoma using apparent diffusion coefficient and diffusion kurtosis indices: preliminary experience in 
fresh liver explants. Magn Reson Imaging, Vol. 30, PP. 1534-1540, 2012.

[34] S Suo, X Chen, X Ji, Investigation of the nonGaussian water diffusion properties in bladder cancer using diffusion kurtosis imaging: a preliminary study. J Comput Assist Tomogr, Vol. 39, PP. 281-285, 2015.

[35] JS Choi, MJ Kim, YE Chung, Comparison of breathhold, navigator-triggered, and freebreathing diffusion-weighted MRI for focal hepatic lesions. J Magn Reson Imaging JMRI, Vol. 38, PP. 109-118, 2013.

[36] M Quentin, G Pentang, L Schimmoller, Feasibility of diffusional kurtosis tensor imaging in prostate MRI for the assessment of prostate cancer: preliminary results. Magn Reson Imaging, Vol. 32, PP. 880-885, 2014.

[37] S Lewis, A Kamath, M Chatterji, Diffusionweighted imaging of the liver in patients with chronic liver disease: comparison of monopolar and bipolar diffusion gradients for image quality and lesion detection. AJR Am J Roentgenol, Vol. 204, PP. 59-68, 2015.
[38] OA Berkhemer, PS Fransen, D Beumer, A randomized trial of intraarterial treatment for acute ischemic stroke. $\mathrm{N}$ Engl $\mathrm{J}$ Med, vo372(1), PP. 11-20, 2015.

[39] TM Leslie-Mazwi, JA Hirsch, GJ Falcone, Endovascular stroke treatment outcomes after patient selection based on magnetic resonance imaging and clinical criteria. JAMA Neurol, Vol. 73(1), PP. 43-49, 2016.

[40] MG Lansberg, VN Thijs, R Bammer, The MRA-DWI mismatch identifies patients with stroke who are likely to benefit from reperfusion. Stroke, Vol. 39(9), PP. 24912496, 2008.

[41] JV Guadagno, EA Warburton, PS Jones, How affected is oxygen metabolism in DWI lesions? a combined acute stroke PETMR study. Neurology, Vol. 67(5), PP. 824-829, 2006.

[42] R Yamada, Y Yoneda, Y Kageyama, K. Ichikawa, Reversal of large ischemic injury on hyper-acute diffusion MRI. Case Rep Neurol, Vol. 4(3), PP. 177-180, 2012. 